\title{
CARACTERÍSTICAS INSTITUCIONAIS DOS PAÍSES E PRÁTICAS DE EVIDENCIACÃO DAS PROVISOOES E PASSIVOS CONTINGENTES AMBIENTAIS: UM ESTUDO INTERNACIONAL
}

Thiago Alberto dos Reis Prado'

Maísa de Souza Ribeiro²

Marcelo Botelho da Costa Moraes ${ }^{3}$

Resumo: Esta pesquisa objetiva investigar a relação entre as características institucionais dos países de origem das empresas, que adotam IFRS, e a evidenciação das provisões e passivos contingentes ambientais. Com base na Teoria Institucional, espera-se que as características institucionais dos países exerçam pressões sobre as práticas de evidenciação, indo de encontro aos objetivos do IASB de comparabilidade em nível global. A pesquisa tem como amostra 614 observações de 123 companhias do Brasil, Canadá, Reino Unido, França, Alemanha, Austrália e China, de setores que exploram o meio ambiente com mais intensidade. O período de análise compreende os anos de 2011 a 2015. Como metodologia, empregou-se análise fatorial e regressões com modelos de dados em painel. Os resultados obtidos mostraram que o disclosure de provisões ambientais está relacionado com o fator construído pelas variáveis de interesse dos sistemas político, financeiro e cultural do país de origem das empresas. No entanto, em relação a passivos contingentes ambientais, não foi encontrada relação estatisticamente significativa com o fator. As evidências de isomorfismo coercitivo e mimético encontradas permitem inferir que a evidenciação de provisões ambientais está relacionada com fatores múltiplos e conflitantes com o escopo de comparabilidade do IASB, o que prejudica este objetivo e sinaliza a não comparabilidade. A principal conclusão deste trabalho é a de que, apesar de existirem pressões normativas para a existência da comparabilidade, há pressões institucionais conflitantes de outros atores sociais, de caráter coercitivo e mimético, fazendo com que as empresas, em busca de legitimidade, conduzam suas práticas de reporte estrategicamente, indo de encontro aos objetivos do IASB.

Palavras-chave: Passivos Ambientais. Provisões Ambientais. Passivos Contingentes Ambientais. Características Institucionais dos Países.

\footnotetext{
thiagoalberto@ufu.br - Universidade Federal de Uberlândia-MG. Brasil. https://orcid.org/0000-0003-1863-3255

${ }^{2}$ imaisorib@usp.br - Universidade de São Paulo-SP. Brasil. https://orcid.org/0000-0003-1165-1813

mbotelho@usp.br - Universidade de São Paulo-SP. Brasil. https://orcid.org/0000-0003-0761-0883

- DOI: http://dx.doi.org/10.14392/asaa.2019120104

- Artigo fruto de Tese de Doutorado de mesmo nome, defendida em Outubro de 2017 na FEA-RP/USP.

- Artigo submetido em: 02/02/2018. Revisões requeridas em: 23/04/2019. Aceito em: 16/07/2019.
} 


\title{
INSTITUTIONAL CHARACTERISTICS OF COUNTRIES AND EVIDENCE PRACTICES OF ENVIRONMENTAL RESERVES ANDLIABILITIES:AN INTERNATIONAL STUDY
}

\begin{abstract}
The aim of this research was to investigate the relationship between institutional characteristics of companies' countries of origin that adopt IFRS and the disclosure of environmental provisions and contingent liabilities. Based on the Institutional Theory, it was expected that the institutional characteristics of countries would exert pressures on disclosure practices, meeting the IASB's objectives of comparability at the global level. The study sampled 614 observations from 123 companies from Brazil, Canada, the United Kingdom, France, Germany, Australia and China, from sectors that exploit the environment with greater intensity. The period of analysis comprises the years 2011 to 2015 . As a methodology, we used factorial analysis and regressions with panel data models. The results showed that the disclosure of environmental provisions is related to the factor constructed by the variables of interest of the political, financial and cultural systems of the country of origin of the companies. However, in relation to environmental contingent liabilities, no statistically significant relationship was found with the factor. The evidence of coercive and mimetic isomorphism found allows us to infer that the disclosure of environmental provisions is related to multiple factors that conflict with the scope of IASB comparability, which undermines this objective and signals non-comparability. The main conclusion of this study is that, although there are normative pressures for the existence of comparability, there are conflicting institutional pressures of other social actors, of coercive and mimetic nature, causing companies, in search of legitimacy, to conduct their practices strategically, contrary to the objectives of the IASB.
\end{abstract}

Keywords: Environmental Liabilities. Environmental Provisions. Environmental Contingent Liabilities. Institutional Characteristics of Countries. 


\section{INTRODUÇÃO}

O brigações de recuperação aos locais degradados durante o ciclo de exploração econômica, bem como penalidades no caso de condutas não ambientalmente responsáveis constituem os passivos ambientais das empresas, e devido às características de incertezas relacionadas à sua existência, ao montante e à data de sua liquidação podem ser consideradas passivos contingentes ou provisões (Chen; Cho \& Patten, 2014).

Passivos ambientais, principalmente em companhias com alto potencial poluidor, podem alcançar quantias relevantes, com impactos expressivos no patrimônio e no resultado das empresas (Baldoíno \& Borba, 2015).

Assim sendo, para Holthausen (1994), indivíduos interessados em avaliar organizações potencialmente sujeitas a custos ambientais devem estimar, com base em informações evidenciadas sobre provisões e passivos contingentes, as implicações destas obrigações no valor das empresas, pois seu reconhecimento afetará o resultado da empresa e sua liquidação impactará as estimativas de fluxos de caixa futuros. A divulgação destas informações também é importante; pois, mesmo que não ocorra o reconhecimento, usuários podem incorporá-las em seus modelos de avaliação e predição (Blacconiere \& Patten, 1994).

O entendimento das consequências da divulgação de informações sobre os passivos ambientais, em conformidade com as normas de reporte, motivou a realização de pesquisas empíricas, as quais sugerem que a divulgação destas informações tem relação positiva com os preços das ações (Barth \& Mcnichols, 1994; Blacconiere \& Northcut, 1997; Campbell, Sefcik \& Soderstrom, 1998), relação negativa com o custo do capital próprio (Garber \& Hammith, 1998) e relação positiva com earnings response coeficientes (Bae \& Sami, 2005).

Os resultados dos estudos indicam também que apesar da relevância das informações relativas aos passivos ambientais e da emissão de normas que exigem sua evidenciação, esta é feita de maneira heterogênea entre as empresas, com poucas informações reportadas em relação ao exigido pelas normas do país (Barth \& Mcnichols, 1994; Cox \& Douthett Junior, 2009).

Evidenciar informações sobre passivos ambientais, principalmente em empresas que causam maiores impactos ambientais, é de suma importância, pois trata-se de gastos que podem ser materiais e que dependem de estimativas para sua mensuração e reconhecimento.

A evidenciação heterogênea entre empresas similares, em desconformidade com as normas de reporte, prejudica a comparabilidade das demonstrações financeiras, que é alcançada quando empresas sujeitas ao mesmo evento econômico reconhecem, mensuram e evidenciam tal evento do mesmo modo (Zeff, 2007).

Na revisão da literatura, encontraram-se poucos estudos que analisaram os fatores que influenciam a evidenciação das provisões e dos passivos contingentes ambientais em conformidade com normas de reporte aplicáveis nos países. Um desses estudos foi o de Barth, McNichols \& Wilson (1997), que analisaram companhias estadunidenses, com o intuito de examinar os fatores relacionados às decisões de evidenciação dos passivos ambientais. Os resultados sugerem que, enforcement da norma SAB 92, materialidade das obrigações ambientais, informações disponibilizadas pela Environmental Protection Agency (EPA), incertezas sobre os valores das obrigações, e o número de locais contaminados que a empresa é responsável estão relacionados com a divulgação. 
Leal, Costa, Oliveira \& Rebouças (2015), em busca de fatores explicativos para as práticas de divulgação, analisaram companhias abertas brasileiras. Os resultados sugerem que a divulgação de informações sobre provisões e passivos contingentes ambientais pelas empresas da amostra é influenciada pelas variáveis: tamanho da empresa, nível de governança, setor de atuação e participação no Índice de Sustentabilidade Empresarial. Adicionalmente, asseveram que motivações externas parecem ser mais significativas para explicar a divulgação e sugerem que estudos futuros poderiam comparar empresas de diferentes países, com o objetivo de encontrar outros fatores explicativos.

Em países que adotam IFRS, o IASB trabalha na convergência das normas contábeis locais a um padrão de âmbito mundial. Neste contexto, características institucionais não deveriam influenciar nas práticas de reporte relativas a provisões e passivos contingentes ambientais, regulamentadas pela norma International Accounting Standard (IAS) no 37, para os países que adotam IFRS. No entanto, segundo Kvaal \& Nobes (2010), mesmo com IFRS, podem haver diferenças em práticas contábeis, pela influência das características dos países na aplicação de normas.

Contudo, não se encontraram, até a realização desta pesquisa, estudos cross-country, que investigassem a conformidade das empresas de diferentes países às práticas de evidenciação aplicáveis para provisões e passivos contingentes ambientais, o que possibilitaria analisar a influência das características dos países nas práticas de reporte.

Assim sendo, o objetivo geral deste estudo é investigar a relação entre características institucionais dos países de origem das empresas, que adotam IFRS, e a evidenciação das provisões e passivos contingentes ambientais.

A pesquisa contribui com estudos sobre adoção de IFRS nos países, por meio da comparação entre práticas e por testar a eficácia da norma em harmonizar o reporte.

Este artigo estrutura-se em cinco seções. Encontra-se, na primeira, a introdução. Na segunda, apresentam-se a revisão da literatura e as hipóteses de pesquisa. Descrevem-se, na terceira seção, os procedimentos metodológicos da pesquisa. Na quarta, há a exposição dos resultados encontrados e sua análise e; na quinta apresentam-se as considerações finais do trabalho, bem como suas limitações e sugestões para estudos futuros.

\section{PLATAFORMA TEÓRICA E HIPÓTESES}

\subsection{Teoria Institucional}

De acordo com Moll, Burns \& Major (2006), a contabilidade é formatada de acordo com o contexto institucional. Assim, sua forma e papel são determinados pelo ambiente organizacional. Neste contexto, segundo os autores, a teoria institucional ganhou relevância nos últimos anos para explicar os fenômenos contábeis.

A nova sociologia institucional é o enfoque que teve mais influência nas pesquisas recentes de contabilidade (Silva, Martins \& Lemes, 2016). Por este motivo ele é tratado com mais detalhes e é o que embasa este estudo. De acordo com ele, as estruturas organizacionais e os procedimentos, incluindo a contabilidade sofrem a influência de fatores externos que, muitas vezes, se sobrepõem à eficiência econômica (Moll et al., 2006). Assim, organizações em ambientes similares são pressionadas a assumirem comportamentos apropriados a estes ambientes, para terem legitimidade.

De acordo com DiMaggio \& Powell (1983), a congruência entre os procedimentos organizacionais e as pressões institucionais é explicada por um processo denominado isomorfismo, em que as organizações se estruturam a partir das exigências de seu ambiente, por meio de práticas isomórficas. $O$ isomorfismo 
institucional pode ser subdividido em três subcategorias, que são: isomorfismo coercitivo; isomorfismo normativo e isomorfismo mimético.

O isomorfismo coercitivo é baseado em influências políticas e legitimidade. Fundamenta que uma organização é influenciada por outra organização, da qual ela é dependente ou ainda de expectativas culturais da sociedade em geral (Dias Filho \& Machado, 2012).

Ainda segundo Dias Filho e Machado (2012), o isomorfismo normativo existe quando as organizações adotam as estruturas e os procedimentos recomendados por associações de profissionais ou consultores, não havendo exigências.

O isomorfismo mimético existe quando organizações imitam as estruturas internas e os procedimentos adotados por outras organizações, que elas consideram como bem-sucedidos (DiMaggio \& Powell, 1983). Quando um claro curso de ação não está disponível, as organizações tendem a mimetizar os pares do mesmo setor ou região, ou de setor e região diferentes (Silva et al., 2016). Essas três formas de isomorfismo representam fontes complementares de pressões acerca das organizações para aderirem aos padrões institucionais (Aerts, Cormier \& Magnan, 2007).

Scott (1995) define que as instituições consistem de elementos regulativos, normativos e culturais e atividades e recursos associados, que fornecem estabilidade e sentido para o comportamento social. Elas são disseminadas por meio de vários mecanismos, tais como culturas, estruturas e rotinas, e operam em múltiplos níveis de jurisdição.

Nesse âmbito, Scott (2005) considera que tais elementos constituem os "Três Pilares das Organizações", e assevera que elas são compostas por várias combinações desses elementos com variações próprias para cada uma, haja vista que eles sustentam as organizações.

Segundo Scott (2005), os isomorfismos do estudo de Dimaggio \& Powell (1983) são responsáveis pela disseminação de cada elemento. Considerando tais colocações, a disseminação de elementos regulativos ocorre por meio de isomorfismo coercitivo; enquanto elementos normativos são disseminados mediante isomorfismo normativo e elementos cultural-cognitivos são disseminados por intermédio de isomorfismo mimético.

No contexto específico da Contabilidade, a homogeneidade das práticas contábeis, almejada pelo IASB em nível global, depende da magnitude das pressões de mecanismos motivadores, em detrimento aos desmotivadores, sendo estes mecanismos caracterizados como isomorfismos coercitivos, normativos e miméticos, por meio da influência do próprio órgão, de outros órgãos de classe, reguladores, legislações contábeis dos países e opinião pública.

Há múltiplas e contraditórias demandas institucionais que as organizações estão sujeitas e elas tenderão a agir de maneira estratégica para responderem às diferentes pressões institucionais, na busca por legitimidade (Scott, 2005).

Algumas destas demandas são elementos normativos, como pressões do IASB e outros órgãos de classe. Logo, há pressões de caráter normativo para a comparabilidade. Por outro lado, há pressões de caráter regulativo e cultural-cognitivo que não contribuem com o objetivo de comparabilidade. Essas pressões, de modo geral, são derivadas das características institucionais dos países, as quais influenciam a opinião pública e o comportamento dos preparadores. Nesse sentido, características heterogêneas conduzem a práticas contábeis heterogêneas (Cieslewicz, 2014).

Neste contexto, a comparabilidade das demonstrações financeiras pode ser um fator secundário, pois o que as empresas almejam, de fato, é a busca por legitimidade e por perspectiva de sobrevivência na sociedade (Souza \& Lemes, 2016). 


\subsection{A relação entre as características institucionais dos países e as práticas de disclosure}

Os resultados de estudos anteriores sugerem que práticas de reporte podem estar relacionadas a características institucionais dos países, de caráter político, financeiro e cultural (Jorgensen \& Soderstrom, 2007; Cieslewicz, 2014; Marino et al., 2016; Alrazi, De Villers \& Van Staden, 2016).

Cieslewicz (2014) assevera que as concepções culturais dos profissionais contábeis e dos usuários, bem como as características das instituições não mudam simplesmente em função da adoção de novas normas contábeis. Dessa forma, leis e guias de aplicações de normas podem ser entendidas de maneira mais flexível por determinada nação, enquanto outras as interpretam de maneira mais rígida e literal, por exemplo. Assim, transações similares podem receber tratamentos contábeis distintos.

Marino et al. (2016) denominam as características institucionais dos países (sistemas político, financeiro, cultural e etc.) de sistemas nacionais de negócios, com base em Whitley (1999). Este assevera que as diferenças de práticas empresariais existentes entre os países podem ser explicadas a partir dos sistemas nacionais de negócios.

Segundo Whitley (1999), o sistema político representa a eficácia dos países em regular direta ou indiretamente os mercados, bem como estabelecer restrições aos comportamentos dos agentes econômicos, por meio de leis e regulamentos.

Leis e regulamentos desempenham um papel importante na facilitação do envolvimento das organizações com o Estado e com seus stakeholders. Consequentemente, em países caracterizados por terem sistemas políticos mais rígidos, há maior conformidade das empresas com os dispositivos legais, para evitar problemas com o Estado e com a opinião pública (Ioannou \& Serafeim, 2012).

Já os sistemas financeiros dos países, de acordo com Whitley (1999), são simplesmente divididos em market-based, em países em que há mercados de capitais grandes e líquidos, e bank-based, em que os mercados de capitais são fracos e ilíquidos. Países de sistema legal de origem common law possuem sistemas financeiros market-based e países de sistema legal de origem code law possuem sistemas financeiros bank-based.

Segundo Ball, Kothari \& Robin (2000), em países common law, a contabilidade sofre mais influência de seus acionistas, que elegem membros para os conselhos de administração, e demandam maiores níveis de disclosure da informação contábil das firmas para a redução da assimetria informacional.

Neste sentido, acredita-se que essas pressões dos sistemas político e financeiro dos países de origem das empresas caracterizam-se como elementos regulativos (Scott, 2005), que utilizam mecanismos de isomorfismo coercitivo (Dimaggio \& Powell, 1983), pelo fato de estarem relacionados à aplicação de leis e regulamentos nos países, além de exigências do mercado de capitais e seus shareholders, aos quais as empresas são dependentes.

Em relação ao sistema cultural, de acordo com Hofstede, "cultura é a programação coletiva da mente que distingue os membros de um grupo humano de outro" (Hofstede, 2011, p.3). Dessa maneira, cada "grupo humano" possui" programações coletivas" distintas, ou seja, existem características culturais diferentes entre os países, no tocante a individualismo, distância do poder e aversão à incerteza, isso forma o sistema cultural (Whitley, 1999). Estas características são enraizadas na mente dos diferentes atores sociais, tais como gestores e contadores e influenciam em suas práticas empresariais (Cieslewicz, 2014).

Assim, acredita-se que os sistemas culturais dos países de origem das empresas caracterizam-se como elementos cultural-cognitivos (Scott, 2005), pois se baseiam em valores internacionalizados nos indivíduos, que influenciam nas interpretações subjetivas necessárias à aplicação de normas contábeis pelos preparadores e nas expectativas informacionais dos usuários. 
Estes elementos não constituem pressões objetivas por meio de regras, regulamentos e recomendações de grupos de profissionais e sim pressões em relação ao que é culturalmente apoiado em determinado ambiente. Dessa forma, em busca de legitimidade, as empresas imitam as práticas de seus pares, que consideram bem-sucedidos no ambiente, à procura de legitimidade, o que constitui mecanismo de isomorfismo mimético (Dimaggio \& Powell, 1983).

O objetivo do estudo de Marino et al. (2016) foi mostrar como as forças que operam no campo institucional afetam o disclosure de Responsabilidade Social Corporativa de companhias do Brasil e Canadá. Os resultados sugerem que há relação entre o disclosure de Responsabilidade Social Corporativa e as proxys para sistemas nacionais de negócios.

Com base no que foi exposto nesta plataforma teórica, foram elaboradas algumas hipóteses, apresentadas na subseção seguinte.

2.3 Hipóteses de pesquisa

H1. Há relação entre as características institucionais dos países de origem das empresas e a evidenciação das provisões ambientais.

H2. Há relação entre as características institucionais dos países de origem das empresas e a evidenciação dos passivos contingentes ambientais.

As proxies utilizadas para características institucionais, bem como as respectivas fontes de coletas, são expostas na seção de procedimentos metodológicos.

\section{PROCEDIMENTOS METODOLÓGICOS}

\subsection{Seleção da amostra e período de estudo}

Os países, companhias, setores econômicos e períodos analisados neste trabalho foram escolhidos de forma não probabilística intencional. Em relação a países, a escolha dos que fariam parte da amostra, obedeceu a quatro critérios: (i) países que adotam normas IFRS; (ii) países com maiores mercados de capitais; (iii) países mais poluidores; e (iv) países com características institucionais heterogêneas. Dessa maneira, optou-se por Brasil, Canadá, Reino Unido, França, Alemanha, Austrália e China.

$\mathrm{Na}$ escolha das empresas, também se procurou analisar aquelas que tivessem maior pressão dos stakeholders pela divulgação. Consequentemente, escolheram-se as empresas componentes do The Dow Jones STOXX Global Total Market Index (DJ STOXXTOTAL) que engloba as companhias responsáveis por $95 \%$ do volume de ações negociadas no mundo.

Em relação aos setores econômicos, optou-se pela escolha de setores que exploram o meio ambiente de maneira mais intensa, os quais consequentemente causam mais degradação (Cormier \& Magnan, 1997). Tais setores são, de acordo com a classificação da DJ STOXX TOTAL, Recursos Básicos, Químico, Construção e Materiais, Óleo e Gás e Utilidades.

O período de análise teve início em 2011, pelo fato de neste ano todas as empresas dos países analisados já reportarem em IFRS. Canadá e China foram os últimos analisados a aderirem às normas IFRS. A adoção obrigatória iniciou-se no ano de 2011. Este período encerrou-se em 2015 para ambos, totalizando 614 observações, de 123 companhias. 
Para a coleta das informações relativas às variáveis dependentes (evidenciação de provisões e passivos contingentes ambientais), empregou-se a técnica análise de conteúdo. Objetiva-se, com a utilização da análise de conteúdo nas notas explicativas das Demonstrações Financeiras anuais publicadas pelas companhias, gerar índices de disclosure para avaliar a conformidade do reporte das companhias em relação à norma IAS 37.

Nessas notas, coletaram-se, para a composição dos índices, as informações que fossem de maneira explícita e exclusiva de provisões e passivos contingentes de natureza ambiental. Estas informações foram coletadas principalmente das notas explicativas relacionadas a provisões e passivos contingentes.

Para cada Demonstração Financeira dos 5 anos do período de análise, atribuíram-se um índice de disclosure de provisões ambientais (DISCPA) e um índice de disclosure de passivos contingentes ambientais (DISCPCA). Tais índices foram obtidos calculando-se a razão entre o número de itens divulgados nas Demonstrações Financeiras de cada empresa e o correspondente número de itens exigidos.

A relação dos itens que cada companhia deve divulgar, para cada classe de provisão e passivo contingente, tais como ambiental, trabalhista e tributária, de acordo com a norma IAS 37, segue no Quadro 1.

Quadro 1 - Divulgação requerida pela norma IAS 37

\begin{tabular}{|l|r|}
\hline \multicolumn{2}{|c|}{ Provisões } \\
\hline 1 & O valor contábil no início e no fim do período \\
\hline 2 & Provisões adicionais feitas no exercício, incluindo aumento nas provisões existentes \\
\hline 3 & Valores utilizados (ou seja, incorridos e baixados contra a provisão) durante o exercício \\
\hline 4 & Valores não utilizados e revertidos durante o período \\
\hline 5 & O aumento durante o período no valor, descontado a valor presente proveniente da passagem do tempo. \\
\hline 6 & Indicação das incertezas sobre o valor ou o cronograma das saídas de benefícios econômicos \\
\hline 7 & Passivos contingentes \\
\hline 1 & Uma breve descrição da natureza do passivo contingente \\
\hline 2 & A estimativa de seu efeito financeiro de acordo com os requisitos de mensuração (melhor estimativa/valor esperado) \\
\hline 3 & Indicação das incertezas relacionadas ao valor ou momento de ocorrência de qualquer saída \\
\hline
\end{tabular}

Obs: A norma também exige a divulgação para provisões do efeito de qualquer mudança na taxa de desconto e do valor de qualquer reembolso esperado, declarando o valor de qualquer ativo que tenha sido reconhecido por conta desse reembolso esperado e, para passivos contingentes, a possibilidade de qualquer reembolso. Contudo, esses itens se enquadram em ambientes e situações bastante específicas, não sendo aplicáveis à maioria das empresas. Por isso não fazem parte dos índices de disclosure. Fonte: Elaborado pelos autores, de acordo com a IAS 37

Neste sentido, para cada divulgação nas Demonstrações Financeiras em conformidade com o que orienta a norma foi atribuído o score 1. Quando a divulgação requerida não existe, atribuiu-se o score 0 . Posteriormente, calculou-se a razão do total de itens divulgados que receberam score 1 e o total de itens requeridos pela IAS 37. Tal metodologia fora adaptada de estudos anteriores (Barth, Mcnichols \& Wilson, 1997; Cox \& Douthett Junior, 2009; Leal et al., 2015).

Para variáveis independentes de controle, empregaram-se características individuais das firmas, com base em estudos anteriores (Barth et al., 1997; Cox \& Douthett Junior, 2009; Leal et al., 2015) e setores econômicos. A relação das variáveis independentes de interesse, características institucionais dos países de origem das empresas, seguem no Quadro 2, com as proxys utilizadas e os sinais esperados. 
Quadro 2 - Relação de variáveis de interesse

\begin{tabular}{|c|c|c|c|c|}
\hline Sistema & $\begin{array}{l}\text { Caracteristicas institucionais } \\
\text { dos países (Interesse) }\end{array}$ & Proxy & Sinal esperado & Fonte de coleta \\
\hline \multirow{4}{*}{ Político (POLI) } & $\begin{array}{l}\text { Proteção a investidores } \\
\text { (PROT) }\end{array}$ & Score do World Bank. & $(+)$ & World Bank \\
\hline & $\begin{array}{l}\text { Enforcement da regulação } \\
\text { contábil (ENRC) }\end{array}$ & $\begin{array}{l}\text { Score baseado em } \\
\text { questionários aplicados a } \\
\text { executivos de vários países. }\end{array}$ & $(+)$ & $\begin{array}{c}\text { The Global Competitiveness } \\
\text { Report }\end{array}$ \\
\hline & $\begin{array}{l}\text { Enforcement da regulação } \\
\text { ambiental (ENRA) }\end{array}$ & $\begin{array}{l}\text { Score baseado em } \\
\text { questionários aplicados a } \\
\text { executivos de vários países. }\end{array}$ & $(+)$ & $\begin{array}{c}\text { The Global Competitiveness } \\
\text { Report }\end{array}$ \\
\hline & $\begin{array}{l}\text { Controle de corrupção } \\
\text { (CONT) }\end{array}$ & Score do World Bank. & $(+)$ & World Bank \\
\hline Financeiro (FINA) & Mercado Financeiro (FIN) & $\begin{array}{l}\text { Variável dicotômica. } 1 \text { para } \\
\text { market-based e } 0 \text { para } \\
\text { bank-based. }\end{array}$ & $(+)$ & La Porta et al., (1997) \\
\hline \multirow{3}{*}{ Cultural (CULT) } & Aversão à incerteza (AVER) & Score de Hofstede. & $(+)$ & $\begin{array}{l}\text { Hofstede, Hofstede \& } \\
\quad \text { Minkov (2010) }\end{array}$ \\
\hline & Distância do poder (DIST) & Score de Hofstede. & $(-)$ & Hofstede et al. (2010) \\
\hline & & Score de Hofstede. & $(+)$ & Hofstede et al. (2010) \\
\hline
\end{tabular}

Fonte: Elaborado pelos autores

\subsection{Técnicas de análise de dados}

Considerando que, neste estudo, foram analisadas 123 companhias abertas em um período de tempo de cinco anos, o modelo de regressão com dados em painel é adequado, pois este consiste em uma combinação de modelo de corte transversal e modelo de séries temporais (Wooldridge, 2010). Destaca-se que estudos anteriores em que se analisou mais de um período também utilizaram essa modelagem (Barth et al., 1997; Cox \& Douthett Junior, 2009; Leal et al., 2015).

Uma crítica a pesquisas que envolvem características institucionais é o número reduzido de variáveis country-level incluídas nos modelos de pesquisa. Uma possível razão é a alta correlação entre as variáveis do país, o que pode causar problemas de colinearidade (Alrazi et al., 2016). No presente estudo, encontrou-se alta correlação entre a maioria das variáveis de interesse, o que implica em problemas de colinearidade, ao incluir todas as variáveis no modelo de regressão. A matriz de correlações é exposta na Tabela 1.

Tabela 1 - Matriz de correlação das variáveis de interesse

\begin{tabular}{|c|c|c|c|c|c|c|c|c|}
\hline & ENRC & ENRA & PROT & CONT & IND & DIST & AVER & FIN \\
\hline ENRC & 1 & & & & & & & \\
\hline ENRA & 0,6756 & 1 & & & & & & \\
\hline PROT & 0,5523 & 0,0746 & 1 & & & & & \\
\hline CONT & 0,8879 & 0,8571 & 0,367 & 1 & & & & \\
\hline IND & 0,8896 & 0,7930 & 0,2595 & 0,9426 & 1 & & & \\
\hline DIST & $-0,8392$ & $-0,8783$ & $-0,3147$ & $-0,8727$ & $-0,8636$ & 1 & & \\
\hline AVER & 0,213 & 0,451 & 0,1024 & 0,4631 & 0,2656 & $-0,4039$ & 1 & 1 \\
\hline FIN & 0,8088 & 0,4607 & 0,4768 & 0,6995 & 0,7953 & $-0,786$ & 0,1582 & 1 \\
\hline
\end{tabular}

Fonte: Dados da pesquisa

Para lidar com o referido problema optou-se por agrupar as variáveis em fatoriais, de acordo com o sistema de negócios. Assim, as sete variáveis de interesse dos sistemas político e cultural foram agrupadas nos fatores POLI e CULT, de acordo com o exposto no Quadro 2. Para o sistema financeiro, não houve necessidade de utilizar fatorial, por tratar-se de apenas uma variável representativa (FINA).

A análise fatorial objetiva descrever um conjunto de variáveis originais por meio da criação de um número menor de variáveis (fatores). A utilização de fatores é aplicável para a obtenção de um conjunto menor de variáveis não correlacionadas para substituir um conjunto de variáveis correlacionadas (Wo- 
oldridge, 2010). Para verificar a adequação da amostra, utilizou-se os testes Kaiser-Meyer-Olkin ( $p$-value 0,0000 e 0,0000) e o Teste de Bartllett (valores de 0,6380 e 0,5220), os quais não apontaram problemas. Posteriormente, estimou-se os scores fatoriais para as variáveis POLI e CULT, com a melhoria da rotação ortogonal.

No entanto, mesmo utilizando a abordagem de sistemas, os problemas de colinearidade permaneceram, impossibilitando a utilização conjunta das variáveis no modelo, conforme a alta correlação exposta na Tabela 2.

Tabela 2 - Matriz de correlação dos fatores

\begin{tabular}{|c|c|c|c|}
\hline & FINA & POLI & CULT \\
\hline FINA & 1 & & \\
\hline POLI & 0,7081 & 1 & \\
\hline CULT & $-0,8191$ & $-0,9549$ & 1 \\
\hline \multicolumn{4}{|c|}{ Fonte: Dados da pesquisa }
\end{tabular}

Assim, optou-se pela utilização de um único fator para as características institucionais dos países, agrupando todas as variáveis de interesse country-level (VARCOUNTRY). O Teste Kaiser-Meyer-Olkin sinalizou p-value 0,0000 e o Teste de Bartllett sinalizou o valor de 0,6470. Posteriormente, estimou-se o score fatorial para a variável VARCOUNTRY, com a melhoria da rotação ortogonal.

Considerando que quase todas as variáveis de interesse apresentam sinal esperado positivo, pelo fato de representarem scores crescentes de rigidez e consequentemente de pressões institucionais dos países de origem das empresas, espera-se sinal positivo também para a variável VARCOUNTRY.

A Equação 1 é a base para o modelo 1, que utiliza DISCPA como variável dependente.

$$
\begin{aligned}
& \text { DISCPA }_{i, t}=\alpha+\beta_{1} \text { RENT }_{i, t}+\beta_{2} \text { MAT }_{i, t}+\beta_{3} \text { TAM }_{i, t}+\beta_{4} \text { END }_{i, t}+\beta_{5} \text { RBAS }_{i}+\beta_{6} \text { OUIM }_{i}+ \\
& \beta_{7} \text { CMAT }_{i}+\beta_{8} \text { OLEO }_{i}+\beta_{9} \text { UTIL }_{i}+\beta_{10} \text { VARCOUNTRY }_{i, t}+\varepsilon_{i, t}
\end{aligned}
$$

A Equação 2 é a base para o modelo 2, que utiliza DISCPCA como variável dependente.

$$
\begin{aligned}
\text { DISCPCA }_{i, t}= & \alpha+\beta_{1} \text { RENT }_{i, t}+\beta_{2} \text { TAM }_{i, t}+\beta_{3} \text { END }_{i, t}+\beta_{4} \text { RBAS }_{i}+\beta_{5} \text { QUIM }_{i}+\beta_{6} \text { CMAT }_{i} \\
& +\beta_{7} \text { OLEO }_{i}+\beta_{8} \text { UTIL }_{i}+\beta_{9} \text { VARCOUNTRY }_{i, t}+\varepsilon_{i, t}
\end{aligned}
$$

Em que,

DISCPAi, $t$ = Índice de Disclosure das provisões ambientais da empresa i no período $t$; DISCPCAi, $t=$ Índice de Disclosure dos passivos contingentes da empresa i no período t; RENT i,t $=$ ROA da empresa i no período $\mathrm{t}$; MAT i,t $=$ materialidade das provisões ambientais da empresa i no período $\mathrm{t} ;$ TAM $\mathrm{i}, \mathrm{t}=$ logaritmo natural dos ativos totais da empresa i no período t; END i,t $=$ endividamento (debt to assets) da empresa i no período t; RBAS i = variável binária que representa que a empresa i pertence ao setor de Recursos Básicos;QUIM i = variável binária que representa que a empresa i pertence ao setor Químico; CMAT i = variável binária que representa que a empresa i pertence ao setor de Construção e Materiais; OLEO i= variável binária que representa que a empresa i pertence ao setor de Óleo e Gás; UTIL $\mathrm{i}=$ variável binária que representa que a empresa i pertence ao setor de Utilidades; VARCOUNTRY $\mathrm{i}, \mathrm{t}=$ Fator das variáveis country-level (de interesse) do país de origem da empresa i no período t; e $\varepsilon$ i,t $=$ termo de erro da regressão.

Wooldridge (2010) assevera que existem três tipos de modelos de dados em painel: pooled, efeitos fixos e efeitos aleatórios. A escolha está sujeita a variações dos dados, o objetivo da pesquisa e a três testes de verificação da consistência dos modelos para os dados analisados, sendo eles Chow, Breush-Pagan e Hausman. 
Considerando o nível de significância de 5\%, os resultados dos testes de Chow e Breush-Pagan mostraram que, para todos os modelos, a estimação pooled não é adequada, existindo efeitos fixos ou efeitos aleatórios. Entre efeitos fixos ou aleatórios, os resultados dos testes de Hausman revelaram que a estimação de efeitos aleatórios é consistente.

O modelo de efeitos aleatórios mostrou-se adequado também na avaliação da variação dos dados e do objetivo da pesquisa e assim foi o utilizado para as análises. Para a verificação da heterocedasticidade, utilizou-se o Teste de White (Wooldridge, 2010). Em todos os modelos encontrou-se a presença de heterocedasticidade, por isso se empregaram erros padrão robustos nos modelos, conforme recomendado por Wooldridge (2010).

Para a verificação de problemas de colinearidade entre as variáveis independentes, utilizou-se o teste de Variance Inflation Factors (VIF). Em nenhuma das variáveis independentes dos modelos do estudo constataram-se problemas de colinearidade. Contudo, o output do software GRETL 2016c omitiu automaticamente a variável setorial CMAT, devido a colinearidade exata com as outras variáveis setoriais.

\section{APRESENTAÇÃO E ANÁLISE DOS RESULTADOS}

\subsection{Estatísticas descritivas}

A Tabela 3 apresenta as estatísticas descritivas das informações referentes às variáveis dependentes DISCPA e DISCPCA de maneira geral e de maneira segregada para cada país de origem das empresas.

Tabela 3 - Estatísticas descritivas por país de origem das empresas

\begin{tabular}{|c|c|c|c|c|c|}
\hline Variável & Média & Mediana & Desvio padrão & Mínimo & Máximo \\
\hline Geral & & & & & \\
\hline DISCPA & 0,6975 & 0,8571 & 0,2634 & 0 & 1 \\
\hline DISCPCA & 0,2271 & 0 & 0,3288 & 0 & 1 \\
\hline Canadá & & & & & \\
\hline DISCPA & 0,8523 & 0,8571 & 0,1985 & 0 & 1 \\
\hline DISCPCA & 0,3361 & 0 & 0,3852 & 0 & 1 \\
\hline Reino Unido & & & & & \\
\hline DISCPA & 0,7129 & 0,8571 & 0,2712 & 0 & 1 \\
\hline DISCPCA & 0,1515 & 0 & 0,2806 & 0 & 1 \\
\hline Austrália & & & & & \\
\hline DISCPA & 0,7413 & 0,8571 & 0,2208 & 0 & 1 \\
\hline DISCPCA & 0,2210 & 0 & 0,3060 & 0 & 0,6667 \\
\hline Alemanha & & & & & \\
\hline DISCPA & 0,7809 & 0,8571 & 0,1274 & 0 & 1 \\
\hline DISCPCA & 0,0833 & 0 & 0,1802 & 0 & 0,6667 \\
\hline França & & & & & \\
\hline DISCPA & 0,7371 & 0,8571 & 0,2127 & 0,1428 & 1 \\
\hline DISCPCA & 0,1111 & 0 & 0,2647 & 0 & 1 \\
\hline Brasil & & & & & \\
\hline DISCPA & 0,4914 & 0,4285 & 0,2902 & 0 & 1 \\
\hline DISCPCA & 0,2666 & 0 & 0,3282 & 0 & 1 \\
\hline China & & & & & \\
\hline DISCPA & 0,4831 & 0,5714 & 0,2048 & 0 & 0,8571 \\
\hline DISCPCA & 0,3939 & 0,6667 & 0,3745 & 0 & 1 \\
\hline & & $F o n t e: D a d o s$ & $0 p e s q u i s a$ & & \\
\hline
\end{tabular}

Fonte: Dados da pesquisa 
Destaca-se, de acordo com a Tabela 3, que o país em que as empresas apresentaram maior média da variável DISCPA foi o Canadá. As empresas do país divulgam em média 85,23\% das informações exigidas.

Todos os países, exceto Brasil e China apresentaram a mediana da variável DISCPA com o valor de 0,8571, o que reflete que grande parte das empresas destes países evidenciam $85,71 \%$ das informações exigidas. As companhias da China e do Brasil apresentaram a menor média e mediana na variável DISCPA, divulgando em média menos de $50 \%$ do exigido, o que sugere que nestes países as companhias possuem menores obrigações ambientais, ou que o ambiente institucional pressiona menos pela evidenciação, em comparação aos demais países analisados.

Aproximadamente metade das companhias brasileiras da amostra, limitaram-se a evidenciar breves descrições das obrigações e seu valor contábil, o que prejudica a comparabilidade com empresas de outros países. As companhias chinesas, em sua maioria, evidenciaram breves descrições das provisões, seu valor contábil e adições. Nenhuma companhia analisada da China reportou a totalidade das informações exigidas.

Em relação a passivos contingentes ambientais, as companhias chinesas foram as que mais evidenciaram informações, com média de 39,39\%. Isso sugere que as empresas do país tendem a considerar mais suas obrigações ambientais como "possíveis" e informar mais os usuários à respeito delas, em relação a outros países da amostra.

Na Alemanha, no Reino Unido e na França, em torno de $80 \%$ das companhias da amostra não divulgam informações sobre seus passivos contingentes ambientais. Isto corrobora com os menores índices médios das empresas destes países, expostos na Tabela 3.

\subsection{Modelo para provisões ambientais}

Na Tabela 4, apresentam-se os outputs do Modelo 1. Como pode ser observado, a variável de interesse VARCOUNTRY possui relação estatisticamente significativa e positiva com a variável DISCPA, conforme esperado.

Tabela 4 - Modelo para provisões ambientais

\begin{tabular}{|c|c|c|}
\hline Variáveis & \multicolumn{2}{|c|}{ Modelo 1 } \\
\hline & Coef. & p-valor \\
\hline RENT & $-0,0740$ & 0,4309 \\
\hline MAT & 0,5399 & $0,0236^{* *}$ \\
\hline TAM & 0,0425 & $0,0026^{* * *}$ \\
\hline END & 0,1235 & $0,0531^{*}$ \\
\hline RBAS & 0,0296 & 0,7369 \\
\hline QUIM & 0,0875 & 0,3463 \\
\hline OLEO & 0,0798 & 0,3897 \\
\hline UTIL & 0,0174 & 0,8507 \\
\hline VARCOUNTRY & 0,1249 & $0,0000^{* * *}$ \\
\hline Observações & 614 & \\
\hline R2 & 0,3100 & \\
\hline Qui-quadrado & 58,3825 & \\
\hline P-valor & 0,0000 & \\
\hline
\end{tabular}

Obs: ***/*** denotam significância bicaudal nos níveis $10 \%, 5 \%$ e 1\%, respectivamente. A variável dependente é DISCPA. Fonte: Dados da pesquisa

O poder explicativo foi de 0,3100. Este número é superior ao encontrado no estudo de Leal et al. (2015), que encontraram 0,1771, e no de Barth et al. (1997), que encontraram 0,2320. Esses estudos não 
utilizaram características institucionais dos países em seus modelos. Os coeficientes das variáveis de controle que apresentaram significância estatística apresentaram sinais conforme o esperado.

Considerando que o coeficiente da variável VARCOUNTRY foi estatisticamente significativo ao nível de $1 \%$ e apresentou sinal positivo, conforme o esperado, pode-se inferir que o disclosure de provisões ambientais está positivamente relacionado com as características institucionais dos países de origem das empresas. Assim, a este nível de significância não se rejeita a hipótese de pesquisa H1.

Isso é um indicativo de que os sistemas político, financeiro e cultural dos países representam pressões institucionais que influenciam nas práticas de reporte, para o alcance de legitimidade. Estes resultados mostram que a homogeneidade das práticas contábeis, que leva à comparabilidade, é prejudicada pelos diferentes níveis de pressões do ambiente em que as companhias operam.

\subsection{Modelo para passivos contingentes ambientais}

Na Tabela 5, apresentam-se os outputs do Modelo 2, que mostram as relações entre a variável dependente DISCPCA, as variáveis controle e a variável de interesse.

Tabela 5 - Modelo para passivos contingentes ambientais

\begin{tabular}{|c|c|c|}
\hline Variáveis & \multicolumn{2}{|c|}{ Modelo 2 } \\
\hline & Coef. & p-valor \\
\hline RENT & $-0,1269$ & 0,5357 \\
\hline TAM & 0,0820 & $0,0059^{* * *}$ \\
\hline END & $-0,0093$ & 0,7997 \\
\hline RBAS & 0,0416 & 0,6254 \\
\hline QUIM & 0,0779 & 0,7141 \\
\hline OLEO & $-0,0627$ & 0,7500 \\
\hline UTIL & 0,0531 & 0,2612 \\
\hline VARCOUNTRY & 0,0422 & \\
\hline Observaçöes & 614 & \\
\hline R2 & 0,190231 & \\
\hline Qui-quadrado & 15,5348 & \\
\hline P-valor & 0,0495429 & \\
\hline P-valor & 0,0000 & \\
\hline
\end{tabular}

Obs: ******* denotam significância bicaudal nos níveis 10\%, 5\% e 1\%, respectivamente. A variável dependente é DISCPCA. Fonte: Dados da pesquisa

Conforme exposto na referida tabela, o coeficiente da variável de interesse não apresentou relação estatisticamente significativa com a variável DISCPCA. O coeficiente da variável TAM foi o único, dentre as variáveis de controle, que apresentou significância estatística a um nível de 1\%.

Portanto, pode-se inferir que a evidenciação dos passivos contingentes ambientais não está relacionada com as características institucionais dos países de origem das empresas. Assim, rejeita-se a hipótese $\mathrm{H} 2$.

Estes resultados mostram que apesar das características institucionais dos países estarem relacionadas às práticas de evidenciação de provisões ambientais, esta relação não existe no tocante a passivos contingentes ambientais.

A heterogeneidade do resultado, em relação ao encontrado para a variável DISCPA, pode ser explicada pelo fato destes passivos contingentes terem, em comparação com as provisões, menor possibilidade de afetarem os fluxos de caixa futuros e estarem sujeitos a maiores incertezas que afetam suas estimativas, não sendo reconhecidas nas Demonstrações Financeiras. Assim, não afetam o resultado, 
nem a estrutura patrimonial das empresas, tendo menor relevância para os usuários (Barth \& Mcnichols, 1994), em comparação com as informações sobre provisões ambientais.

\section{CONSIDERAÇÕES FINAIS}

O objetivo do presente trabalho foi investigar a relação entre as características institucionais dos países de origem das empresas, que adotam IFRS, e a evidenciação das provisões e dos passivos contingentes ambientais.

Como metodologia, utilizaram-se as variáveis DISCPA e DISCPCA como variáveis dependentes e como variáveis independentes as características das empresas, apontadas em outros estudos sobre determinantes do disclosure de provisões e passivos contingentes e um fator que agrupa as características institucionais dos países de origem das empresas, dos sistemas político, financeiro e cultural, apontadas em estudos sobre determinantes cross-country de disclosure em modelos de regressão com dados em painel.

Os resultados dos modelos de dados em painel apontaram que a variável dependente DISCPA apresentou relação estatisticamente significativa e positiva, ao nível de 1\%, com a variável de interesse. Isso sugere a existência de isomorfismo coercitivo e mimético (Dimaggio \& Powell, 1983).

Estes resultados corroboram com os encontrados em estudos que analisaram a relação das características institucionais dos países e práticas de disclosure voluntário (Jorgensen \& Soderstrom, 2007; Marino et al., 2016; Alrazi et al., 2016) e demonstram que características institucionais também influenciam em práticas de reporte exigidas por normas IFRS, prejudicando o objetivo do IASB de comparabilidade em nível global.

Já a variável dependente DISCPCA não apresentou relação estatisticamente significativa com a variável de interesse. A referida variável apresentou relação estatisticamente significativa apenas com o tamanho da empresa, o que sugere que as pressões institucionais dos países não influenciam o reporte de informações sobre passivos contingentes ambientais, ao contrário do que ocorre com as provisões ambientais.

Assim como encontrado no estudo de Alrazi et al. (2016), em relação à evidenciação ambiental voluntária, os achados neste estudo fornecem evidências de que a evidenciação obrigatória de itens ambientais depende das características institucionais dos países de origem das empresas, refutando os argumentos dos defensores de regulamentação da evidenciação ambiental para o alcance da comparabilidade.

Como consequência dos resultados da pesquisa, é possível inferir que apenas regulamentar não é suficiente para harmonizar o reporte, é necessário que o ambiente institucional em que a companhia se insere incentive a evidenciação, evitando a retenção de informações relevantes pela gestão da empresa.

Como principais contribuições, a pesquisa identificou que apesar de existirem pressões normativas do IASB para a existência da comparabilidade, há outras pressões institucionais de caráter regulativo e cultural-cognitivo, as quais são múltiplas e conflitantes e estão relacionadas com as características das empresas e de seus países de origem, e fazem com que as empresas, em busca de legitimidade, conduzam suas práticas de reporte de maneira estratégica para responderem às diferentes pressões institucionais. Isso prejudica a qualidade das informações evidenciadas sobre passivos ambientais e vai de encontro ao objetivo do IASB, de comparabilidade em nível global.

Os resultados deste estudo são relevantes para reguladores, investidores e credores, pois constatou-se a heterogeneidade nas informações divulgadas, influenciada por pressões institucionais, o que 
além de impactar na comparabilidade, também compromete a confiabilidade das informações, pelo fato do que é divulgado depender do contexto em que a companhia se insere.

Como toda pesquisa científica, esta apresenta limitações. As limitações presentes neste trabalho são as seguintes: a) a amostra do estudo é não-probabilística. Esse fato impede a generalização de resultados a outras empresas, países e setores econômicos; b) é possível que algumas companhias tenham sido penalizadas em seus índices de disclosure por não divulgar itens que na verdade não são aplicáveis a elas, por suas particularidades e; c) existe o viés intrínseco ao processo de coleta de dados nas notas explicativas, por meio da técnica de análise de conteúdo, haja vista que ela depende do conhecimento do pesquisador sobre o assunto.

Por fim, o pesquisador assevera que, assim como se identificaram neste estudo evidências de pressões culturais, decorrentes das características institucionais dos países, influenciando em práticas de evidenciação de provisões e passivos contingentes ambientais, é possível que estas pressões também influenciem nas práticas de reporte de outras informações contábeis exigidas e isso deve ser pesquisado em estudos futuros.

Ademais, a pesquisa investigou apenas práticas de evidenciação, mas é possível, pelos pressupostos da Teoria Institucional, que as características institucionais pesquisadas também influenciem nas práticas de reconhecimento e mensuração e isso também deve ser investigado em estudos futuros.

\section{REFERÊNCIAS}

Aerts, W., Cormier, D., \& Magnan, M. (2007). The association between web-based corporate performance disclosure and financial analyst behaviour under different governance regimes. Corporate Governance: An International Review, 15(6), 1301-1329. DOI: 10.1111/j.1467-8683.2007.00648.x

Alrazi, B., De Villiers, C., \& Van Staden, C. J. (2016). The environmental disclosures of the electricity generation industry: a global perspective. Accounting and Business Research, 46(6), 665-701. DOI: 10.1080/00014788.2015.1135781

Bae, B., \& Sami, H. (2005). The effect of potential environmental liabilities on earnings response coefficients. Journal of Accounting, Auditing \& Finance, 20(1), p.43-70. DOI: 10.1177/0148558X0502000103

Baldoíno, E., \& Borba, J. A. (2015) Passivos Contingentes na Bolsa de Valores de Nova York: uma análise comparativa entre as empresas estrangeiras. Revista de Contabilidade e Organizações, 9(23), p. 58-81. DOI: $10.11606 /$ rco.v9i23.68395

Ball, R., Kothari, S. P., \& Robin, A. (2000). The effect of international institutional factors on properties of accounting earnings. Journal of accounting and economics, 29(1), 1-51. DOI: 10.1016/S01654101(00)00012-4

Barth, M.E., \& Mcnichols, M.F. (1994). Estimation and market valuation of environmental liabilities relating to superfund sites. Journal of Accounting Research, 32, p. 177-209. DOI: 10.2307/2491446

Barth, M. E., Mcnichols, M. F.; \& Wilson, G. P. (1997). Factors influencing firms' disclosures environmental liabilities. Review of Accounting Studies, 2, p. 35-64. DOI: 10.1023/A:1018321610509

Blacconiere, W. G., \& Patten, D. M. (1994). Environmental disclosures, regulatory costs, and changes in firm value. Journal of accounting and economics, 18(3), 357-377. DOI: 10.1016/0165-4101(94)90026-4

Blacconiere, W. G., \& Northcut, W. D. (1997). Environmental information and market reactions to environmental legislation. Journal of Accounting, Auditing \& Finance, 12(2), 149-178. DOI: $10.1080 / 10807039.2016 .1247256$ 
Campbell, K.; Sefcik, S. E., \& Soderstrom, N. S. (1998). Site uncertainty, allocation uncertainty, and superfund liability valuation. Journal of Accounting and Public Policy, 17(4), 331-367. DOI: 10.1016/ S0278-4254(98)10009-1

Chen, J., Cho, C.; \& Patten, D. (2014). Initiating Disclosure of Environmental Liability Information: An Empirical Analysis of Firm Choice. Journal of Business Ethics, 125, p. 681-692. DOI: 10.1007/s10551-013-1939-0

Cieslewicz, J. K. (2014). Relationships between national economic culture, institutions, and accounting: Implications for IFRS. Critical perspectives on accounting, 25(6), 511-528. DOI: 10.1016/j.cpa.2013.03.006

Cormier, D., \& Magnan, M. (1997). Investors' assessment of implicit environmental liabilities: an empirical investigation. Journal of Accounting and Public Policy. 16, p. 215-241. DOI: 10.1016/S02784254(97)00002-1

Cox, C. A., E. B. Douthett Junior. (2009). Further evidence on the factors and valuation associated with the level of environmental liability disclosures. Academy of Accounting and Financial Studies Journal, 13(3), p. 1-26.

Dias Filho, J. M., \& Machado, L. H. B. (2004). Abordagens da pesquisa em contabilidade. Teoria avançada da contabilidade. São Paulo: Atlas, 15-69.

DiMaggio, P., \& Powell, W. W. (1983). The iron cage revisited: Collective rationality and institutional isomorphism in organizational fields. American sociological review, 48(2), 147-160. DOI: 10.2307/2095101

Garber, S., \& Hammitt, J. K. (1998) Risk premiums for environmental liability: Does Superfund increase the cost of capital?. Journal of Environmental Economics and Management, 36(3), 267-294. DOI: 10.1006/ jeem.1998.1046

Hofstede, G. (2011). Dimensionalizing cultures: The Hofstede model in context. Online readings in psychology and culture, 2(1), 8. DOI: 10.9707/2307-0919.1014

Holthausen, R. W. (1994). Discussion of estimation and market valuation of environmental liabilities relating to superfund sites. Journal of Accounting Research, 211-219. DOI: 10.2307/2491447

loannou, I., \& Serafeim, G. (2012). What drives corporate social performance? The role of nation-level institutions. Journal of International Business Studies, 43(9), 834-864. DOI: 10.1057/jibs.2012.26

Jorgensen, B. N., \& Soderstrom, N. S. (2007). Environmental disclosures within legal and accounting contexts an international perspective. Columbia business school. Chazen Web Journal, 15.

Kvaal, E., \& Nobes, C. (2010). International differences in IFRS policy choice: a research note. DOI: 10.5539/ ijbm.v12n2p27

Leal, P. H., Costa, B. M., Oliveira, M. C., \& Rebouças, S. M. D. P.

(2015). Divulgação de Provisões e Passivos Contingentes Ambientais sob a Ótica da Teoria Institucional. In: IX Congresso Anpcont. Curitiba: PR.

Marino, P. B. L. P. M., Soares, R. A.; Abreu, M. C.; Rebouças, S. M. D. P. (2016). Efeito do ambiente institucional do Brasil e Canadá na evidenciação da responsabilidade social corporativa. In: XIX Semead. São Paulo.

Moll, J., Burns, J., \& Major, M. (2006). Institutional theory. Methodological issues in accounting research: Theories, methods and issues, 183-205.

Scott, W. R. (1995). Introduction: institutional theory and organizations. The institutional construction of organizations, 11-23.

Silva, D. M., Martins, V. A., \& Lemes, S. (2016). Escolhas Contábeis: reflexões para a pesquisa. Revista Contemporânea de Contabilidade, 13(29). DOI: 10.5007/2175-8069.2016v13n29p129 
Souza, F.E.A., \& Lemes, S. (2016). A comparabilidade das escolhas contábeis na mensuração subsequente de ativos imobilizados, de ativos intangíveis e de propriedades para investimento em empresas da América do Sul. Revista Contabilidade \& Finanças-USP, 27(71). DOI: 10.1590/1808-057x201501480

Whitley, R. (1999). Divergent capitalisms: The social structuring and change of business systems. OUP Oxford.

Wooldridge, J. M. (2010). Econometric analysis of cross section and panel data. MIT press.

Zeff, S. A. (2007). Some obstacles to global financial reporting comparability and convergence at a high level of quality. The British Accounting Review, 39(4), 290-302. DOI: 10.1016/j.bar.2007.08.001 